\title{
Aloe-emodin-mediated photodynamic therapy induces autophagy and apoptosis in human osteosarcoma cell line MG-63 through the ROS/JNK signaling pathway
}

\author{
PINGHUA TU*, QIU HUANG* , YUNSHENG OU, XING DU, KAITING LI, YONG TAO and HANG YIN \\ Department of Orthopedics, The First Affiliated Hospital of Chongqing Medical University, \\ Yuzhong, Chongqing 400016, P.R. China
}

Received December 6, 2015; Accepted February 12, 2016

DOI: $10.3892 /$ or.2016.4703

\begin{abstract}
The present study was carried out to investigate the effect and mechanisms of aloe-emodin (AE)-mediated photodynamic therapy (AE-PDT) on the human osteosarcoma cell line MG-63. After treatment with AE-PDT, the human osteosarcoma cell line MG-63 was tested for levels of viability, autophagy, reactive oxygen species (ROS) and apoptosis and changes in cell morphology with the Cell Counting Kit-8 (CCK-8), monodansylcadaverine (MDC) and Hoechst staining and transmission electron microscopy. The expression of proteins including LC-3, cleaved caspase-3, Beclin-1, Bcl-2, p-JNK, t-JNK and $\beta$-actin was examined with western blotting. AE-PDT significantly inhibited the viability of the MG-63 cells in an AE-concentration- and PDT energy densitydependent manner. Autophagy and apoptosis of MG-63 cells was substantially promoted in the AE-PDT group compared to the control group, the $\mathrm{AE}$ alone group and the light emitting diode (LED) alone group. Inhibition of autophagy by 3-methyladenine (3-MA) $(5 \mathrm{mM})$ and chloroquine (CQ) $(15 \mu \mathrm{M})$ significantly promoted the apoptosis rate and improved the sensitivity of the MG-63 cells to AE-PDT. AE-PDT was found to induce the expression of ROS and p-JNK. ROS scavenger, $N$-acetyl-L-cysteine (NAC, $5 \mathrm{mM}$ ), was able to hinder the autophagy, apoptosis and phosphorylation of JNK, and JNK inhibitor (SP600125, $10 \mu \mathrm{M}$ ) significantly inhibited the autophagy and apoptosis, and attenuated the sensitivity
\end{abstract}

Correspondence to: Dr Yunsheng Ou, Department of Orthopedics, The First Affiliated Hospital of Chongqing Medical University, 1 You Yi Road, Yuanjiagang, Yuzhong, Chongqing 400016, P.R. China E-mail: ouyunsheng2001@163.com

*Contributed equally

Abbreviations: PDT, photodynamic therapy; ROS, reactive oxygen species; AE, aloe-emodin; AE-PDT, aloe-emodin-mediated photodynamic therapy; TCM, traditional Chinese medicine; LED, light emitting diode; TEM, transmission electron microscope

Key words: aloe-emodin, photodynamic therapy, osteosarcoma, autophagy, apoptosis of MG63 cells to AE-PDT. In conclusion, AE-PDT induced the autophagy and apoptosis of human osteosarcoma cell line MG-63 through the activation of the ROS-JNK signaling pathway. Autophagy may play a protective role during the early stage following treatment of AE-PDT.

\section{Introduction}

Osteosarcoma is the most common tumor among malignant tumors in children, and mainly occurs during childhood and adolescence $(1,2)$. Currently, the combination of surgery and auxiliary chemotherapy significantly benefits to improve the survival of patients, but is not ideal for patients suffering recurrent, metastatic or drug-resistant osteosarcoma $(3,4)$. As a new promising approach for treating solid tumors (5-7), photodynamic therapy (PDT) enables the absorption of a photosensitizer to produce reactive oxygen species (ROS) which kills tumor cells and facilitates local photoradiation therapy (8). These studies suggest that PDT plays an important role in treating tumors; however, the related mechanism is not clear.

Aloe-emodin (AE) is a novel anthraquinone compound extracted from traditional Chinese medicine (TCM) plants and has been verified to have antitumor effects $(9,10)$. However, the genotoxicity (e.g. gene lesion and gene mutation) limits its application in large dosage (11). Furthermore, AE has been found to have a certain fluorescence and can be used as a photosensitizer $(12,13)$. The combination of light emitting diode (LED) and AE-mediated photodynamic therapy (AE-PDT) can reduce the $\mathrm{AE}$ concentration to induce the apoptosis of tumor cells and therefore reduce its toxicity $(14,15)$. The present study aimed to investigate the effect of AE-PDT on human osteosarcoma cell line MG-63 and to explore the related mechanisms.

\section{Materials and methods}

Cell culture. The human osteosarcoma cell line MG-63 [purchased from the American Type Culture Collection (ATCC); Manassas, VA, USA] was incubated in Dulbecco's modified Eagle's medium (DMEM) supplemented with $10 \%$ fetal bovine serum (FBS) (both from Gibco, Carlsbad, CA, USA), $100 \mathrm{IU} / \mathrm{ml}$ penicillin and $100 \mu \mathrm{g} / \mathrm{ml}$ streptomycin 
at $37^{\circ} \mathrm{C}$ in a humidified atmosphere of $5 \% \mathrm{CO}_{2}$. When the confluency reached $70 \%$, the cells were divided into a control group (no treatment), an AE group (treatment with only AE), a LED alone group (treatment with only photoradiation) and an AE-PDT group (treatment with AE and photoradiation). For some experiments, the cells were pretreated with ROS inhibitor $\mathrm{N}$-acetyl-L-cysteine (NAC) (5 mM; Beyotime, China), or JNK inhibitor SP600125 (10 $\mu$ M; Sigma, St. Louis, MO, USA). AE at different concentrations $(0,5,10,20$ and $50 \mu \mathrm{M}$; Sigma) was added to the culture medium as indicated for $6 \mathrm{~h}$ and thereafter rinsed 3 times with normal medium. The PDT was applied with a LED light source at $430 \mathrm{~nm}$ of wavelength, continuous input model and density of $40 \mathrm{~mW} / \mathrm{cm}^{2}$ at different radiation periods of $0,60,120$ and $160 \mathrm{sec}$ aiming to produce energy density of $0,2.4,4.8$ and $6.4 \mathrm{~J} / \mathrm{cm}^{2}$, respectively.

Measurement of the cell viability by $C C K-8$. The cells were inoculated in 96-well plates at the density of $5 \times 10^{3}$ cells/well for $24 \mathrm{~h}$, and 3 repetitive wells were prepared for each group. After the corresponding treatments, the cells were cultured for another $12 \mathrm{~h}$ and then treated with $10 \mu \mathrm{l}$ Cell Counting Kit- 8 (CCK-8) (Beyotime) in an incubator for $1 \mathrm{~h}$. The absorption values (A) of CCK-8 [optical density (OD)] at $450 \mathrm{~nm}$ were measured by a microplate reader to calculate the cell growth inhibition rate (\%) according to the formulation: Inhibition rate $(\%)=\left(\mathrm{OD}_{\text {blank }}-\mathrm{OD}_{\text {experiment }}\right) / \mathrm{OD}_{\text {blank }} \times 100 \%$. Based on the inhibition rate, an $\mathrm{AE}$ concentration of $10 \mu \mathrm{M}$ and energy density of PDT at $4.8 \mathrm{~J} / \mathrm{cm}^{2}$ were selected for subsequent experiments.

Measurement of intracellular ROS. The cells were inoculated in 24-well plates at the density of $5 \times 10^{4}$ cells/well. Following corresponding treatments, the cells were incubated with $200 \mu \mathrm{l}$ DCFH-DA (Sigma) at a concentration of $10 \mu \mathrm{M}$ at $37^{\circ} \mathrm{C}$ for $20 \mathrm{~min}$. After rinsing with phosphate-buffered saline (PBS) for 3 times, the cells were digested using $0.25 \%$ trypsin and re-suspended in PBS, and the intensity of fluorescence was measured by flow cytometry according to the manual.

Monodansylcadaverine (MDC) staining. After the corresponding treatment, the cells were rinsed with PBS for 3 times and incubated in $200 \mu \mathrm{l}$ of $0.05 \mathrm{mM}$ MDC (Sigma) at $37^{\circ} \mathrm{C}$ for $30 \mathrm{~min}$. Then, the cells were rinsed with PBS for 2 times and the aggregation of autophagic vacuoles was observed under a fluorescence microscope (DMI4000 B; Leica Microsystems, Wetzlar, Germany) with an excitation wavelength of $460-500 \mathrm{~nm}$ and an emission wavelength of 512-542 nm.

Hoechst 33342 staining. Following the corresponding treatment, cells incubated in 24-well plates were stained with Hoechst ( $10 \mu \mathrm{M}$; Sigma) staining at $37^{\circ} \mathrm{C}$ in the dark for $5 \mathrm{~min}$, and subsequently observed under a fluorescence microscope (DMI4000 B) with an excitation wavelength of 355-366 nm and an emission wavelength of 465-480 nm.

Transmission electron microscope (TEM). After the different treatments, cells collected in EP tubes were centrifuged, fixed with $2.5 \%$ glutaraldehyde and $1 \%$ osmic acid, dehydrated with gradient ethanol and acetone, embedded and sliced, and stained with 3\% uranyl acetate-lead citrate. Finally, the cells were observed with TEM.

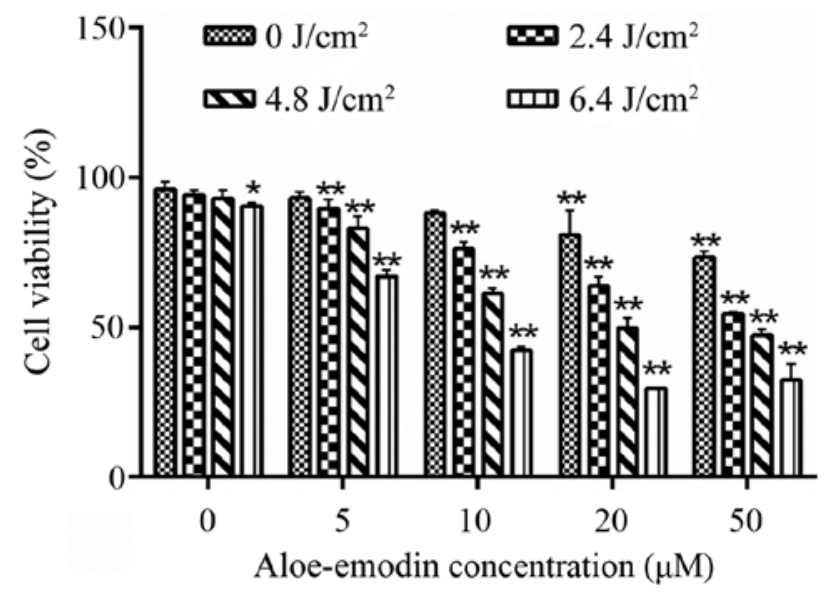

Figure 1. Viability of the MG-63 cells following treatment with different concentrations of aloe-emodin (AE) and energy densities of photodynamic therapy (PDT). MG-63 cells were treated with different concentrations of AE $(0,5,10,20$ and $50 \mu \mathrm{M})$ for $6 \mathrm{~h}$, and then irradiated with various light doses $\left(0,2.4,4.8\right.$ and $\left.6.4 \mathrm{~J} / \mathrm{cm}^{2}\right)$. At $12 \mathrm{~h}$ after irradiation, cell viability was tested by CCK- 8 assay. ${ }^{*} \mathrm{p}<0.05$ vs. the control group; ${ }^{* *} \mathrm{p}<0.01$ vs. the control group.

Measurement of apoptosis by flow ctyometry. After treatment, the MG-63 cells incubated at $1 \times 10^{6}$ cells/well into a 6 -well plate were then stained with Annexin V/propidium iodide (PI) (KeyGen Biotech, China) for flow cytometry, according to the manual.

Western blotting. The collected cells were rinsed with PBS and lysed. An equal volume of protein lysates was added for sodium dodecyl sulfate-polyacrylamide gel electrophoresis (SDS-PAGE). The proteins were transferred to nitrocellulose membranes, blocked with Tris-buffered saline and Tween-20 (TBST) containing 5\% skimmed milk at room temperature for $1 \mathrm{~h}$, rinsed and incubated with the corresponding primary antibodies $\beta$-actin $(1: 1,000)$, Bcl-2 (1:1,000), total-JNK $(1: 1,000)$, p-JNK (1:1,000), cleaved caspase-3 (1:1,000) (all from Cell Signaling Technology, Danvers, MA, USA), Beclin-1 $(1: 1,000)$ and LC-3 $(1: 1,000)$ (both from Sigma) at $4^{\circ} \mathrm{C}$ overnight. After rinsing with TBST, the proteins were incubated with secondary antibodies at room temperature for $1 \mathrm{~h}$. The film was developed with an enhanced chemiluminescence (ECL) detection system.

Statistical analysis. All data are presented as the mean \pm standard deviation (SD). Data were compared with one- or two-way ANOVA for intergroup data and the SNK-q test for intragroup. The differences were considered significant if $\mathrm{p}<0.05$.

\section{Results}

Cell viability of the human osteosarcoma cell line MG-63. The measurement of cell viability by CCK- 8 indicated that $\mathrm{AE}$ alone at the concentration of $0-10 \mu \mathrm{M}$ had no significant inhibition on the viability of MG-63 cells ( $p>0.05$ ). In contrast, cell viability was significantly reduced following treatment of $\mathrm{AE}$ at a concentration of $>10 \mu \mathrm{M}$ (p<0.05; Fig. 1). Similarly, LED alone with an energy density of $0-4.8 \mathrm{~J} / \mathrm{cm}^{2}$ revealed no obvious effect on the viability of the MG-63 cells ( $>0.05$ ). 

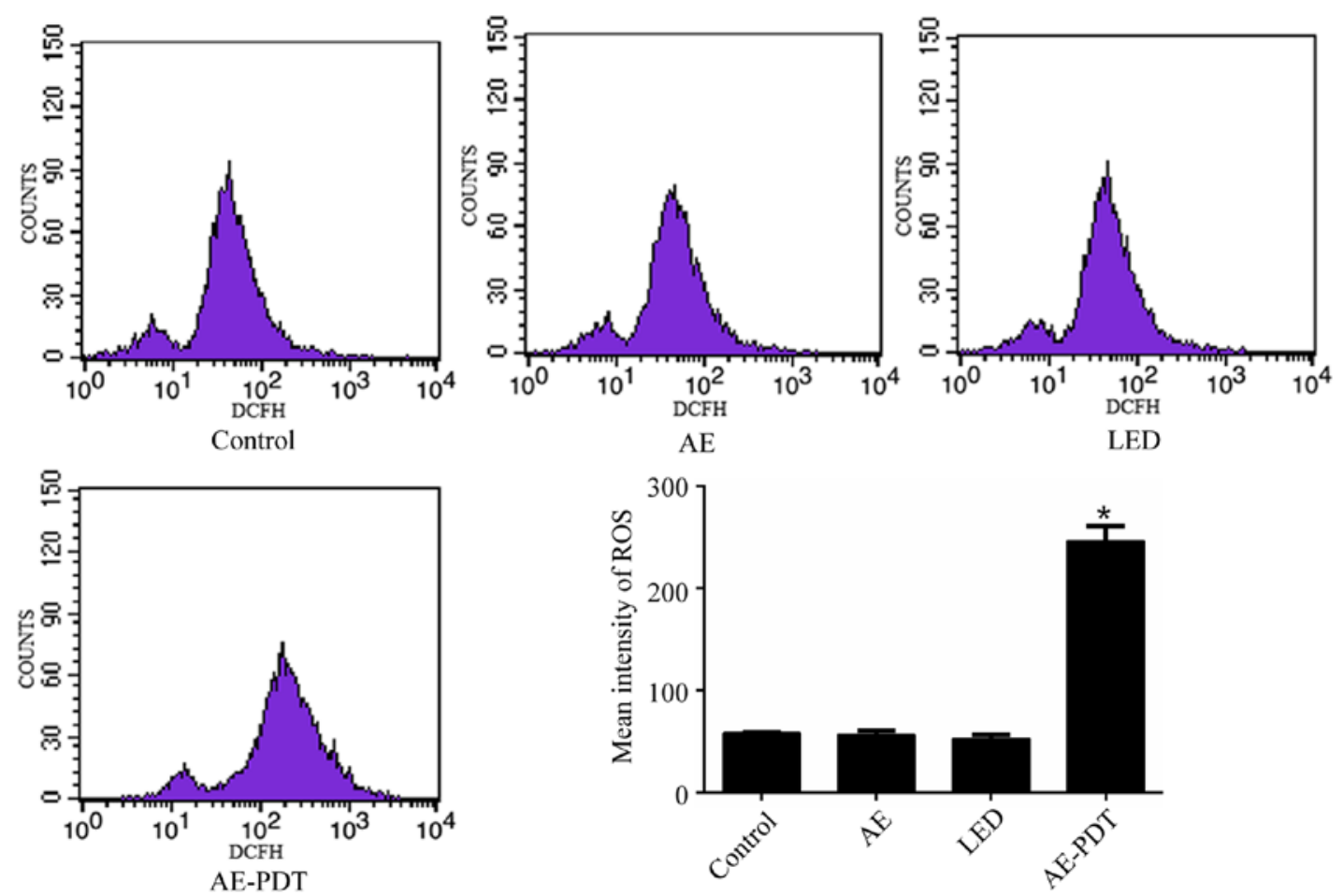

Figure 2. Cellular ROS in the different groups measured by flow cytometry. MG- 63 cells were treated with $10 \mu \mathrm{M}$ aloe-emodin (AE) for $6 \mathrm{~h}$ and then irradiated with light $\left(4.8 \mathrm{~J} / \mathrm{cm}^{2}\right)$, at $3 \mathrm{~h}$ after irradiation. The ROS level was then measured by flow cytometry. ${ }^{*} \mathrm{p}<0.05$ vs. the control group; AE and LED group.

When the LED energy density was $>4.8 \mathrm{~J} / \mathrm{cm}^{2}$, the viability of the MG-63 cells was substantially inhibited (p<0.05; Fig. 1). Furthermore, AE-PDT caused significant inhibition of the viability of MG-63 cells in a dose-dependent manner ( $\mathrm{p}<0.01$; Fig. 1). Therefore, we chose $\mathrm{AE}$ at $10 \mu \mathrm{M}$ and PDT with an energy density of $4.8 \mathrm{~J} / \mathrm{cm}^{2}$ for the subsequent experiments.

Intracellular ROS. Flow cytometry demonstrated that there was no significant difference in the intracellular ROS level between the LED alone group, AE alone group and control group ( $>0.05)$. However, the ROS level in the AE-PDT group was substantially increased when compared to the LED alone group, $\mathrm{AE}$ alone and control group, respectively ( $\mathrm{p}<0.05$; Fig. 2).

Cell morphologic changes. After staining with MDC under a fluorescence microscope, the autophagic vacuoles displayed green spots mainly distributed in the perineuclei. There were no significant changes between the control, AE alone and LED alone group. However in the AE-PDT group, the MG-63 cellular nuclei were surrounded by the aggregation of massive autophagic vacuoles, and a significant difference was found compared with the control, AE alone and LED alone group (Fig. 3).

For Hochest 33342 staining, the MG-63 cells demonstrated a higher density of nuclear chromatin showing highlight blue, obvious pycnosis, aggregation and rapture resulting in typical apoptotic bodies after treatment with AE-PDT (Fig. 4), and these cell changes were increased with an increase in the treatment periods. However, no significant changes occurred in the control, AE alone and LED alone group.

Under TEM, the treatment of MG-63 cells using AE-PDT contributed to produce massive vesicular structure wrapping cellular organelles or protein which was fused with lysosomes to form typical autophagic lysosomes. The autophagic lysosomes were obviously different from necrotic and apoptotic cells and were significantly increased in the AE-PDT group when compared to the control, AE alone group, and LED alone group (red arrow, Fig. 5). Noticeably, there were numerous expanded endoplastic reticulum in the AE-PDT group (hollow arrow, Fig. 5).

Apoptosis and cell viability. When compared to the control group, the apoptosis rate in the AE alone and LED alone group was not significantly different ( $>00.05$; Fig. 6A). However, the apoptosis rate in the AE-PDT group was substantially increased and higher relative to that in the control group, $\mathrm{AE}$ alone and LED alone group ( $<<0.05$; Fig. 6A). Pretreatment with $5 \mathrm{mM} 3$-MA (AE-PDT+3-MA group) and $15 \mu \mathrm{M}$ chloroquine (CQ) (AE-PDT+CQ group) significantly increased the apoptosis rate ( $\mathrm{p}<0.05$; Fig. $6 \mathrm{~A})$ and promoted cell death when compared to the AE-PDT group ( $<<0.05$; Fig. 6B).

Expression of apoptosis and autophagy-related protein. The results indicated that the AE alone and LED alone treatments had no significant influence on the expression of apoptotic and autophagic proteins including LC-3II, cleaved caspase-3, Beclin-1, Bcl-2 and p-JNK (p>0.05; Fig. 7). However, AE-PDT was able to significantly increased the expression of LC-3II, cleaved caspase-3, Beclin-1 and p-JNK in a time-dependent manner ( $\mathrm{p}<0.05$; Fig. 7).

Effects of NAC and SP600125 on autophagy, apoptosis and cell viability. The pretreatment of cells with ROS inhibitor NAC $(5 \mathrm{mM})$ was found to inhibit the expression of apoptotic 


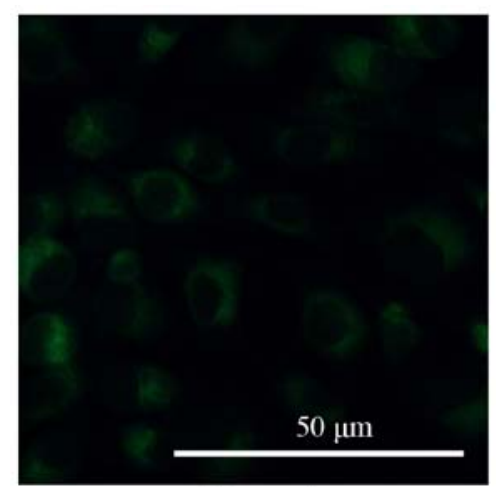

Control

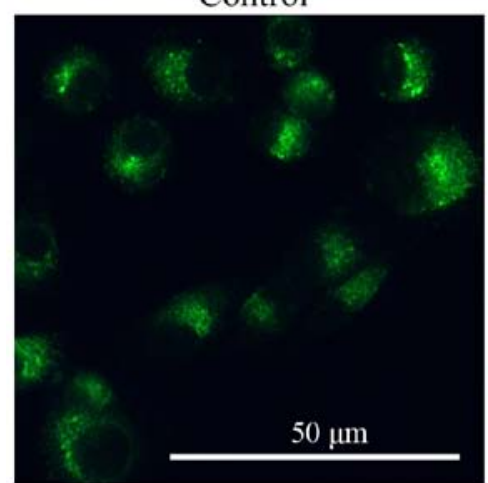

AE-PDT $3 \mathrm{~h}$

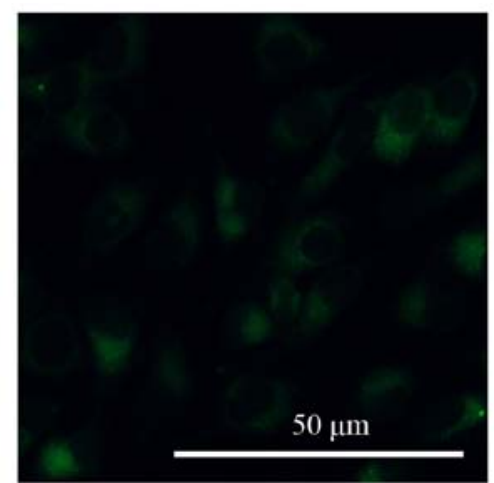

$\mathrm{AE}$

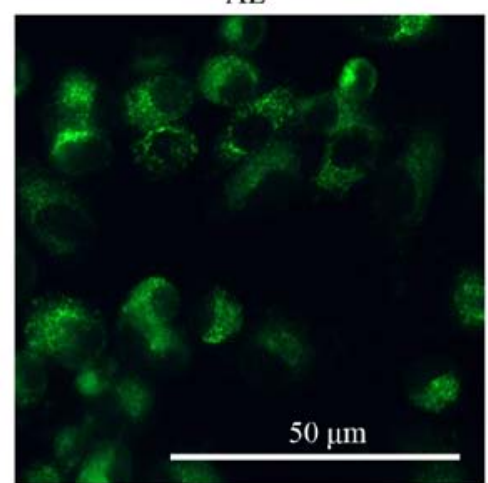

AE-PDT $6 \mathrm{~h}$

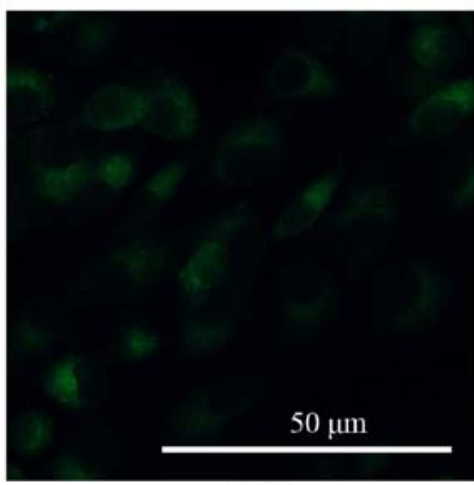

LED

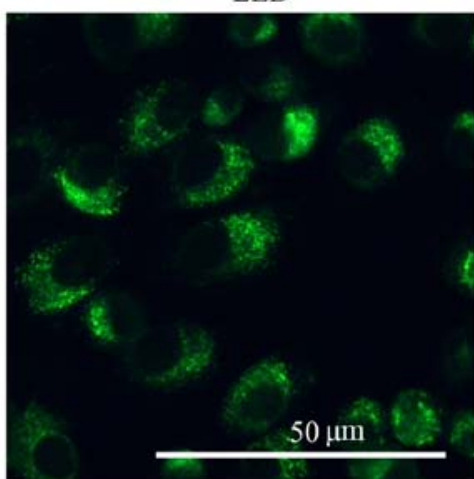

AE-PDT $12 \mathrm{~h}$

Figure 3. Autophagic morphological changes were evaluated by fluorescence microscopy using monodansylcadaverine (MDC) staining. MG-63 cells were treated with $10 \mu \mathrm{M}$ aloe-emodin (AE) for $6 \mathrm{~h}$ and then irradiated with light $\left(4.8 \mathrm{~J} / \mathrm{cm}^{2}\right)$. At 3,6 and $12 \mathrm{~h}$ after irradiation, autophagic vacuoles were detected using MDC staining.

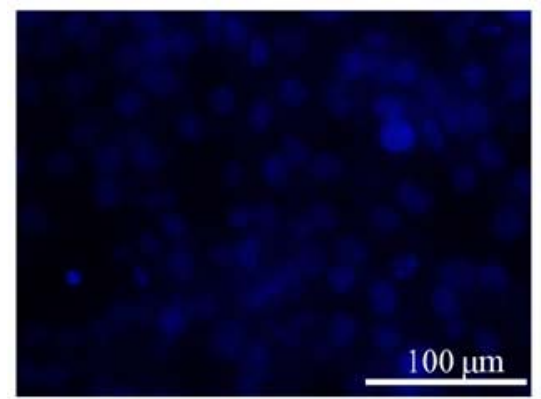

Control

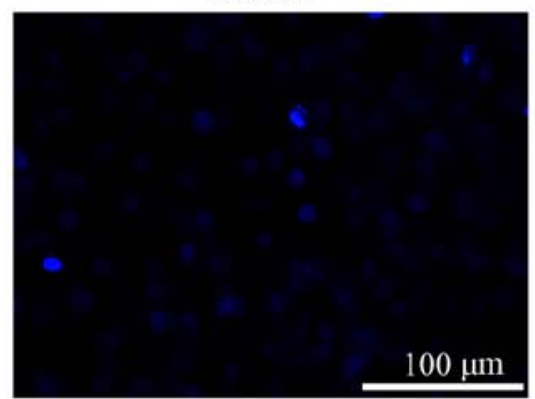

AE-PDT $3 \mathrm{~h}$

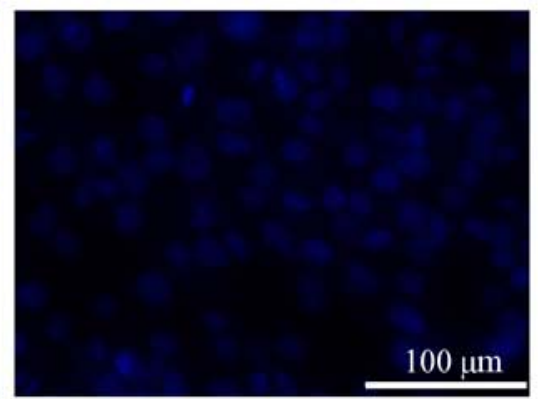

$\mathrm{AE}$

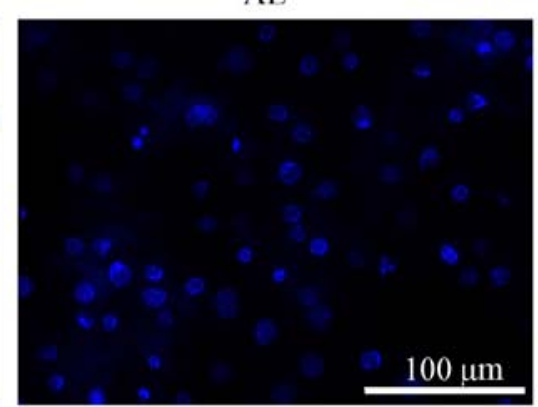

AE-PDT $6 \mathrm{~h}$

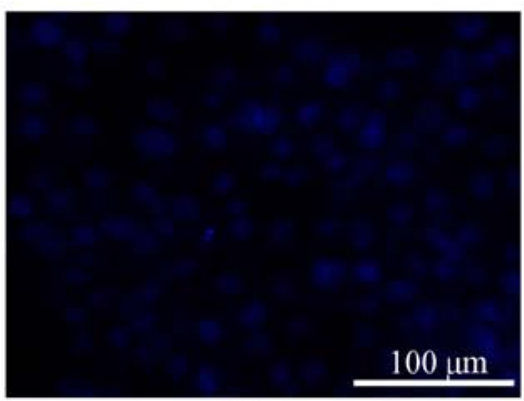

LED

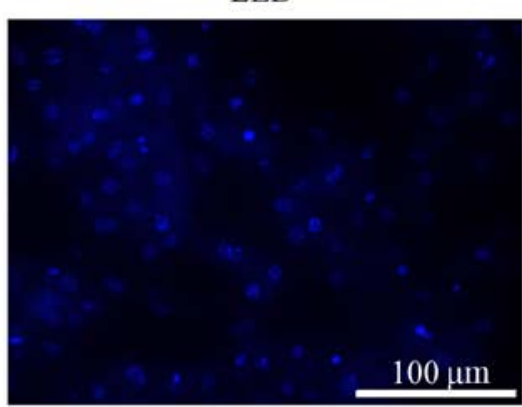

AE-PDT 12 h

Figure 4. Apoptotic morphological changes were evaluated by fluorescence microscopy using Hoechst 33342 staining. MG- 63 cells were treated with $10 \mu \mathrm{M}$ aloe-emodin (AE) for $6 \mathrm{~h}$ and then irradiated with light $\left(4.8 \mathrm{~J} / \mathrm{cm}^{2}\right)$. At 3, 6 and $12 \mathrm{~h}$ after irradiation, apoptotic cells were observed by Hochest staining.

and autophagic proteins p-JNK, cleaved caspase-3 and LC3-II followed by the treatment of AE-PDT $(p<0.05$; Fig. 8A). Similarly, the pretreatment of cells with JNK inhibitor
SP600125 $(10 \mu \mathrm{M})$ significantly reduced the expression of apoptotic and autophagic proteins p-JNK, cleaved caspase-3 and LC3-II followed by the treatment of AE-PDT $(p<0.05$; 


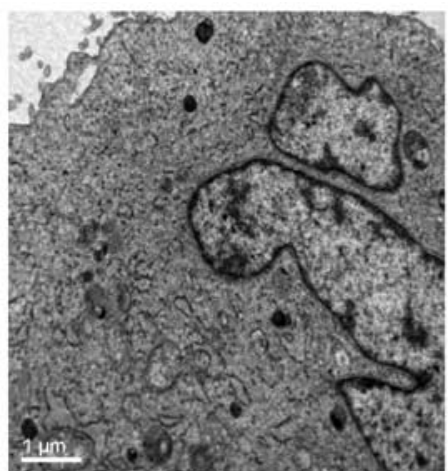

Control

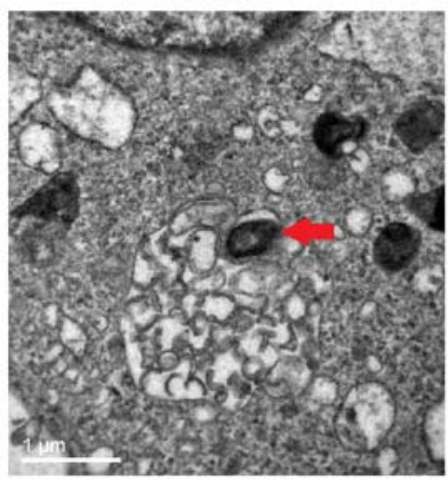

AE-PDT $3 \mathrm{~h}$

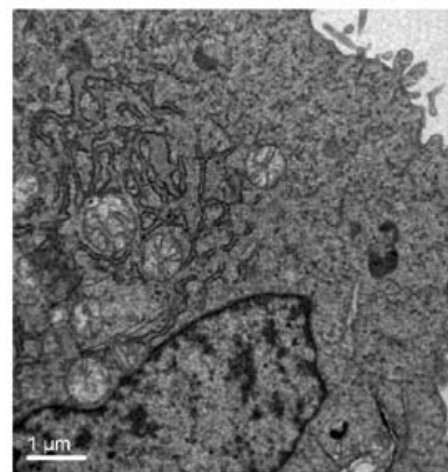

$\mathrm{AE}$

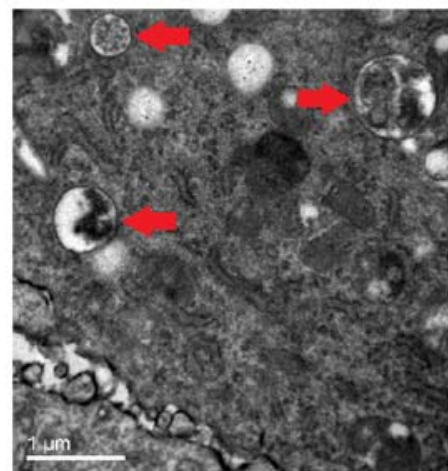

AE-PDT $6 \mathrm{~h}$

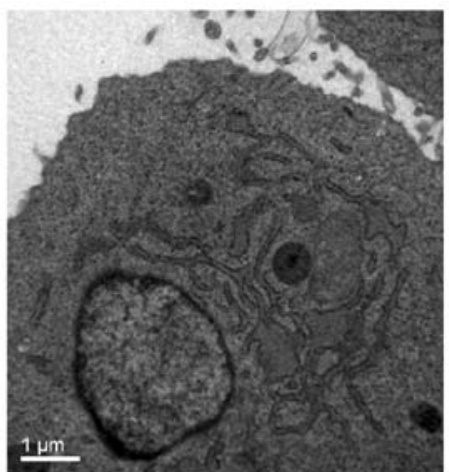

LED

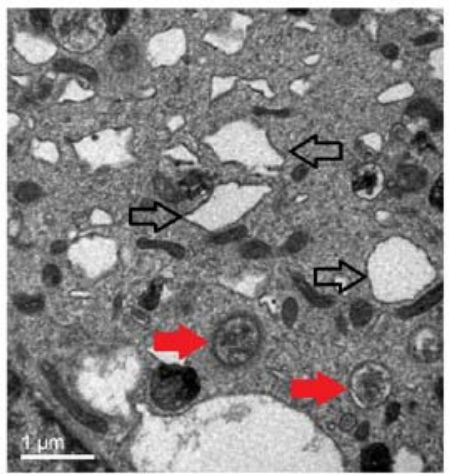

AE-PDT $12 \mathrm{~h}$

Figure 5. Transmission electron microscopy (TEM) was utilized to evaluate the changes in autophagosomes. MG-63 cells were treated with $10 \mu \mathrm{M}$ aloeemodin (AE) for $6 \mathrm{~h}$ and then irradiated with light $\left(4.8 \mathrm{~J} / \mathrm{cm}^{2}\right)$. At 3,6 and $12 \mathrm{~h}$ after irradiation, autophagosomes were observed by TEM.

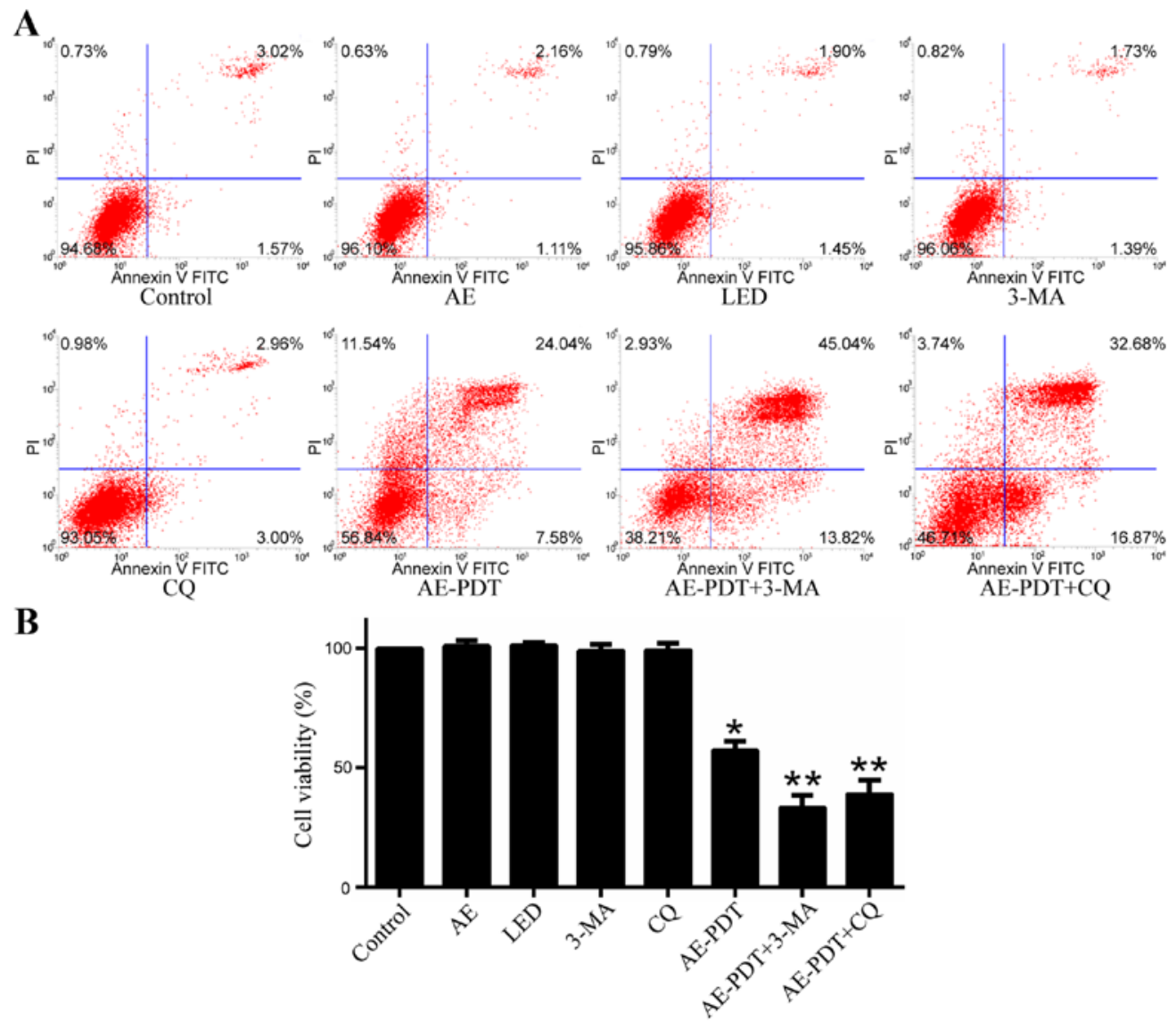

Figure 6. Apoptosis and cell viability in the different groups were measured by flow cytometry and CCK-8 assays. After the corresponding treatments (A) MG-63 cells were stained with Annexin V-PI and analyzed by flow cytometry. (B) Cell viability was determined by CCK-8 assay. "p $<0.05$ vs. the control group; ${ }^{* *}$ p $<0.05$ vs. the AE-PDT group. 


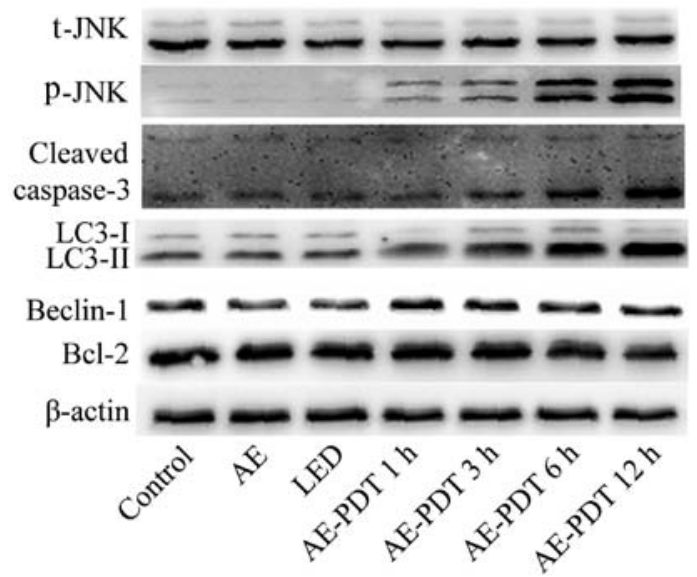

Figure 7. Expression of apoptosis and autophagy-related proteins in the MG-63 cells following AE-PDT treatment. MG-63 cells were treated with $10 \mu \mathrm{M}$ aloe-emodin (AE) for $6 \mathrm{~h}$ and then irradiated with light $\left(4.8 \mathrm{~J} / \mathrm{cm}^{2}\right)$. At 1, 3, 6 and $12 \mathrm{~h}$ after irradiation, the expression levels of t-JNK, p-JNK, cleaved caspase-3, LC3, Beclin-1 and Bcl-2 were assessed by western blotting.

Fig. 8B). Furthermore, the pretreatment with SP600125 $(10 \mu \mathrm{M})$ significantly increased the viability of the MG-63 cells when compared to that noted in the AE-PDT group ( $\mathrm{p}<0.05$; Fig. $8 \mathrm{C}$ ).

\section{Discussion}

PDT is a newly developed invasive therapeutic approach for the treatment of tumors by utilizing visible light at a certain wavelength to excite a photosensitizer aggregated around tumors. After excitation, the photosensitizer can induce a series of photochemical and photobiological reactions to produce ROS, further damaging subcellular organelles, inducing cell death and causing antitumor effects (8). Although PDT is a promising therapeutic strategy for solid tumors, the molecular mechanism of PDT is still not completely clear.

$\mathrm{AE}$ is known as an active extract of traditional Chinese herbs, and has fluorescence and an important antitumor effect with dose-dependent cytotoxicity (11-15). In the present study, only $\mathrm{AE}$ at a concentration higher than $10 \mu \mathrm{M}$ showed potential in inducing the apoptosis of human osteosarcoma MG-63 cells, suggesting that a high concentration of $\mathrm{AE}$ is required for antitumor therapy. However, its dosage for clinical application must be reduced due to genotoxicity leading to gene lesions and gene mutations (11). A previous study indicated that $\mathrm{AE}$ alone at the concentration of $50 \mu \mathrm{M}$ has the ability to promote the apoptosis of pulmonary carcinoma, while AE-PDT (AE at a concentration of $20 \mu \mathrm{M}$ and PDT at an energy density of $1.6 \mathrm{~J} /$ $\mathrm{cm}^{2}$ ) showed a comparable antitumor effect on lung cancer cell line H460 (15). In the present study, the results indicated that AE-PDT (AE at a concentration of $10 \mu \mathrm{M}$ and PDT at an energy density of $4.8 \mathrm{~J} / \mathrm{cm}^{2}$ ) significantly reduced the viability of human osteosarcoma MG-63 cells, and triggered autophagy and apoptosis. Thus, these studies suggested that AE-PDT has a significant antitumor effect at a low dosage by utilizing the photosensitizing features of $\mathrm{AE}$, and at the same time reducing the related side-effects triggered by a higher concentration of drugs.

The ROS-JNK pathway is involved in AE-PDT-induced autophagy and apoptosis. ROS signaling plays an important

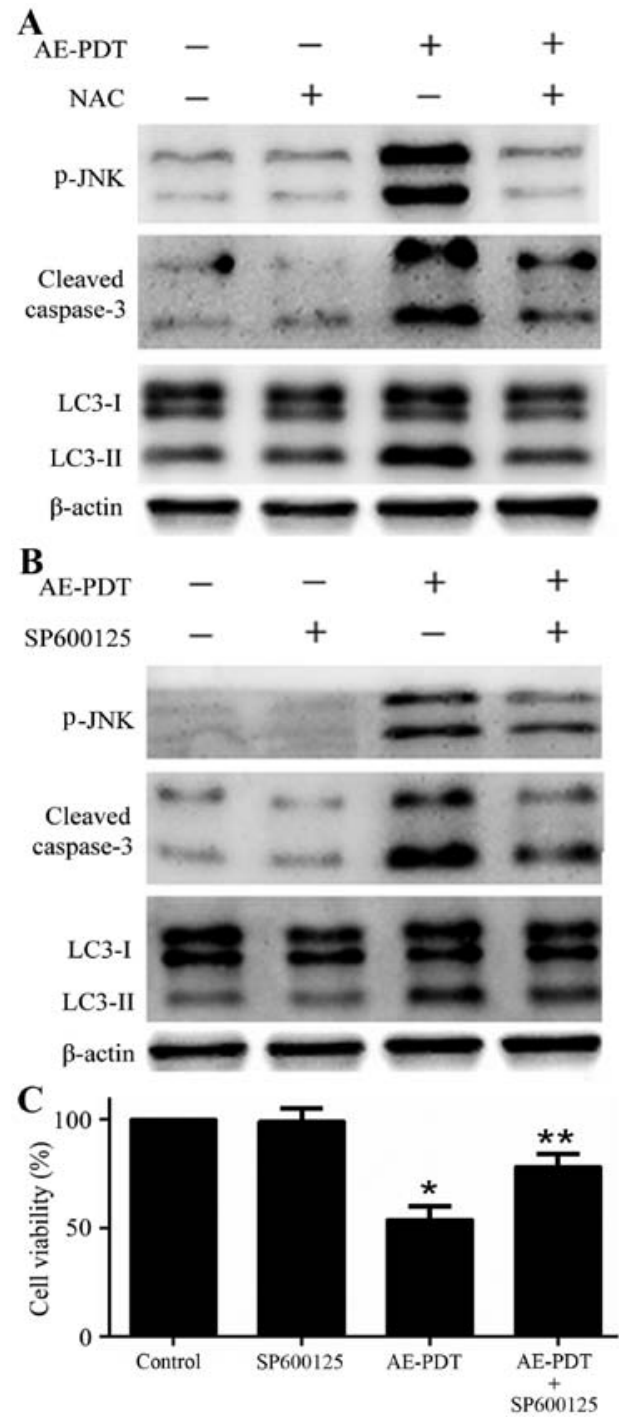

Figure 8. Effects of NAC and SP600125 on autophagy, apoptosis and cell viability of the AE-PDT-treated MG-63 cells. * $\mathrm{p}<0.05$ vs. the control group; ${ }^{* *} \mathrm{p}<0.05$ vs. the AE-PDT group. (A) MG-63 cells were treated without or with AE-PDT in the presence or absence of NAC (5 mM), and $12 \mathrm{~h}$ later, the expression of p-JNK, cleaved caspase-3 and LC3 was detected by western blotting. (B) MG-63 cells were treated without or with AE-PDT in the presence or absence of SP600125 $(10 \mu \mathrm{M})$. Twelve hours later, the expression of p-JNK, cleaved caspase-3 and LC3 was detected by western blotting. (C) Cell viability was evaluated by CCK- 8 .

role in PDT and a low dosage of ROS contributes to improve cellular function and cell survival. In contrast, excessive ROS cause the capability to promote programmed cell death (16). The present study indicated that the ROS level in the human osteosarcoma MG-63 cells treated with AE-PDT was significantly increased and cleaved caspase- 3 was also gradually increased. ROS inhibitor NAC was found to prevent these changes, suggesting that ROS were involved in the apoptosis induced by AE-PDT. Following stress stimulus, ROS can induce the formation of autophagosome, and previous studies indicate that ROS play a critical role in promoting autophagy by activating the JNK pathway $(17,18)$. The present study revealed that AE-PDT significantly activated the JNK pathway in MG-63 cells while inhibition of ROS prevented the activation of JNK, suggesting that ROS were the proximal event of JNK. Furthermore, the JNK inhibitor was found to partially 
block the expression of autophagic and apoptotic proteins, and reverse the AE-PDT-induced inhibition of cell viability. Based on these data, we conclude that JNK was involved in the AE-PDT-induced autophagy and apoptosis in MG-63 cells and acted as a downstream of ROS, and thus the mechanism of the AE-PDT-mediated antitumor effect was associated with ROS-JNK interaction. This finding is consistent with some recent studies suggesting that ROS-induced JNK activation plays an important role in inducing tumor cell apoptosis and autophagy $(16,19)$.

Autophagy can protect tumor cells through the cleavage of ROS and inhibition of apoptosis while in some cases it can render the autophagic death of tumor cells and contribute to tumor suppression $(20,21)$. This suggests that autophagy plays a dual roles in antitumor effects. Synthesized pheophorbide a-mediated PDT was found to hold some promise in killing oral squamous carcinoma cells through inducing apoptosis and autophagy, and inhibition of autophagy enhanced the apoptosis (22). These results suggest that autophagy plays a role in PDT-mediated antitumor therapy. This hypothesis was supported by the finding that pretreatment of MG-63 cells with 3-MA, a specific inhibitor of the early stage autophagic process (23), and CQ, a late stage autophagy inhibitor (23), significantly increased the apoptosis rate, and further reduced the cell viability when compared to the AE-PDT group. Therefore, we propose that autophagy may have a protective effect during the early stage of AE-PDT.

In summary, the present study indicated that AE-PDT effectively decreased the viability of human osteocarcinoma MG-63 cells in an AE concentration- and PDT energy density-dependent manner, and induced autophagy and apoptosis through activation of the ROS-JNK signaling pathway. Furthermore, autophagy may play a protective role during the early stage of AE-PDT. Further research is needed to enhance the antitumor effect of AE-PDT by modulating autophagy and apoptosis.

\section{Acknowledgements}

The present study was supported by the National Natural Science Foundation of China (81572634), the Graduate Scientific Innovation Project of Chongqing Education Committee (CYS14122 and CYS15141) and the Technologic and Scientific Development Program of Chongqing Science and Technology Committee (CSTC2012gg-yyjs10018). The authors wish to thank Mr. Yong Zhu, Mr. Bin He and Mr. Zenghui Zhao, of the Department of Orthopedics, The First Affiliated Hospital of Chongqing Medical University, Chongqing, China, for their advice and supervision with regards to the statistical analysis and modification of the manuscript.

\section{References}

1. Whelan J, McTiernan A, Cooper N, Wong YK, Francis M, Vernon S and Strauss SJ: Incidence and survival of malignant bone sarcomas in England 1979-2007. Int J Cancer 131: E508-E517, 2012.

2. Ottaviani G and Jaffe N: The epidemiology of osteosarcoma. Cancer Treat Res 152: 3-13, 2009.
3. He H, Ni J and Huang J: Molecular mechanisms of chemoresistance in osteosarcoma (Review). Oncol Lett 7: 1352-1362, 2014.

4. Mittal N, Kent PM and Ording J: Metastatic and recurrent bone primary bone cancers. Curr Probl Cancer 37: 215-224, 2013.

5. Shirasu N, Nam SO and Kuroki M: Tumor-targeted photodynamic therapy. Anticancer Res 33: 2823-2831, 2013.

6. Calabrò G, Patalano A, Lo Conte V and Chianese C: Photodynamic chemotherapy in the treatment of superficial mycoses: An evidence-based evaluation. G Ital Dermatol Venereol 148: 639-648, 2013.

7. Master A, Livingston M and Sen Gupta A: Photodynamic nanomedicine in the treatment of solid tumors: Perspectives and challenges. J Control Release 168: 88-102, 2013.

8. Mfouo-Tynga I and Abrahamse H: Cell death pathways and phthalocyanine as an efficient agent for photodynamic cancer therapy. Int J Mol Sci 16: 10228-10241, 2015.

9. Chen R, Zhang J, Hu Y, Wang S, Chen M and Wang Y: Potential antineoplastic effects of Aloe-emodin: A comprehensive review. Am J Chin Med 42: 275-288, 2014

10. Tabolacci C, Lentini A, Mattioli P, Provenzano B, Oliverio S, Carlomosti $\mathrm{F}$ and Beninati S: Antitumor properties of aloeemodin and induction of transglutaminase 2 activity in B16-F10 melanoma cells. Life Sci 87: 316-324, 2010.

11. Sevcovicova A, Bodnarova K, Loderer D, Imreova P, Galova E and Miadokova E: Dual activities of emodin - DNA protectivity vs mutagenicity. Neuro Endocrinol Lett 35 (Suppl 2): S149-S154, 2014.

12. Zaffaroni M, Mucignat C, Pecere T, Zagotto G, Frapolli R, D'Incalci $M$ and Zucchetti M: High-performance liquid chromatographic assay for the determination of Aloe Emodin in mouse plasma. J Chromatogr B Analyt Technol Biomed Life Sci 796: 113-119, 2003.

13. Vargas F, Rivas C and Medrano M: Interaction of emodin, aloeemodin, and rhein with human serum albumin: A fluorescence spectroscopic study. Toxicol Mech Methods 14: 227-231, 2004.

14. Lee HZ: Effects and mechanisms of emodin on cell death in human lung squamous cell carcinoma. Br J Pharmacol 134: 11-20, 2001.

15. Lee HZ, Yang WH, Hour MJ, Wu CY, Peng WH, Bao BY, Han PH and Bau DT: Photodynamic activity of aloe-emodin induces resensitization of lung cancer cells to anoikis. Eur J Pharmacol 648: 50-58, 2010.

16. Trachootham D, Lu W, Ogasawara MA, Nilsa RD and Huang P: Redox regulation of cell survival. Antioxid Redox Signal 10: 1343-1374, 2008.

17. Rodríguez-Blanco J, Martín V, García-Santos G, Herrera F, Casado-Zapico S, Antolín I and Rodriguez C: Cooperative action of JNK and AKT/mTOR in 1-methyl-4-phenylpyridinium-induced autophagy of neuronal PC12 cells. J Neurosci Res 90: 1850-1860, 2012.

18. Erikstein BS, Hagland HR, Nikolaisen J, Kulawiec M, Singh KK, Gjertsen BT and Tronstad KJ: Cellular stress induced by resazurin leads to autophagy and cell death via production of reactive oxygen species and mitochondrial impairment. J Cell Biochem 111: 574-584, 2010.

19. Zou P, Xia Y, Chen T, Zhang J, Wang Z, Chen W, Chen M, Kanchana K, Yang S and Liang G: Selective killing of gastric cancer cells by a small molecule targeting ROS-mediated ER stress activation. Mol Carcinog: Jun 18, 2015 (Epub ahead of print). doi: $10.1002 / \mathrm{mc} .22351$.

20. Gewirtz DA: The four faces of autophagy: Implications for cancer therapy. Cancer Res 74: 647-651, 2014.

21. Bincoletto C, Bechara A, Pereira GJ, Santos CP, Antunes F, Peixoto da-Silva J, Muler M, Gigli RD, Monteforte PT, Hirata H, et al: Interplay between apoptosis and autophagy, a challenging puzzle: New perspectives on antitumor chemotherapies. Chem Biol Interact 206: 279-288, 2013

22. Ahn MY, Yoon HE, Kwon SM, Lee J, Min SK, Kim YC, Ahn SG and Yoon JH: Synthesized Pheophorbide a-mediated photodynamic therapy induced apoptosis and autophagy in human oral squamous carcinoma cells. J Oral Pathol Med 42: 17-25, 2013.

23. Abe A and Kokuba H: Harmol induces autophagy and subsequent apoptosis in U251MG human glioma cells through the downregulation of survivin. Oncol Rep 29: 1333-1342, 2013. 